\title{
ON POLYNOMIAL INVARIANTS OF VIRTUAL LINKS
}

\author{
V. O. MANTUROV
}

\begin{abstract}
The $V A$-polynomial proposed in the author's earlier paper (Acta Appl. Math. 72 (2002), 295-309) for virtual knots and links is considered in this paper. One goal here is to refine the definition of this polynomial to the case of the ring $\mathbb{Z}$ in place of the field $\mathbb{Q}$. Moreover, the approach in the paper mentioned makes it possible to recognize "long virtual knots" obtained from equivalent virtual knots by cutting at various points. An invariant of long virtual knots that is based on the same technique as the $V A$-polynomial is proposed. Some properties of the $V A$-polynomial are established. Furthermore, new invariants of virtual links and long virtual knots are constructed.
\end{abstract}

\section{Contents}

$\S 1$. Introduction

$\S 2$. Construction of the $V A$-polynomial and some of its properties

$\S 3$. How is the $V A$-polynomial computed?

$\S 4$. Table of values of the $V A$-polynomial

$\S 5$. Some properties of the $V A$-polynomial

$\S 6$. The case of $\mathbb{Z}\left[t, t^{-1}\right]$

$\S 7$. The polynomial $\mathcal{V}$

\section{$\S 1$. INTRODUCTION}

The theory of virtual knots was proposed by Kauffman in 1996 (see [3]). It originates from the theory of knots in thickened surfaces $S_{g} \times I$, which was first studied by Kauffman, Jaeger, and Saleur (see [2]). Virtual knots arise upon projecting $S_{g}$ on $\mathbb{R}^{2}$, and hence $S_{g} \times \mathbb{R}$ on $\mathbb{R}^{3}$. In projecting link diagrams from $S_{g}$ on $\mathbb{R}^{2}$, we obtain planar diagrams. Virtual crossings arise as defects of the projection, that is, points of intersection of the images of arcs that do not intersect in $S_{g}$. At the very beginning of the study of this theory its creators proposed a generalization of the basic knot invariants: the distributive groupoid, the fundamental group, and the Jones-Kauffman polynomial (see [3]). On the other hand (see [1]), virtual knots appear in connection with the nonrealizability of certain Gaussian diagrams as diagrams of classical knots (see also [4]).

A virtual link diagram is defined to be a 4-valent graph imbedded in the plane in which each crossing is labeled as classical (with an indication of which branch is an overcrossing and which an undercrossing) or as virtual, in which case a small circle is put at the crossing.

According to 3, a virtual link is an equivalence class of virtual link diagrams with respect to generalized Reidemeister moves. Generalized Reidemeister moves consist of the three classical Reidemeister moves (in which only classical crossings take part), the three purely virtual analogues of the classical Reidemeister moves (in which only virtual crossings take part), and a single "semivirtual move" shown in Figure 1.

2000 Mathematics Subject Classification. Primary 57M27, 57M25; Secondary 12E10. 


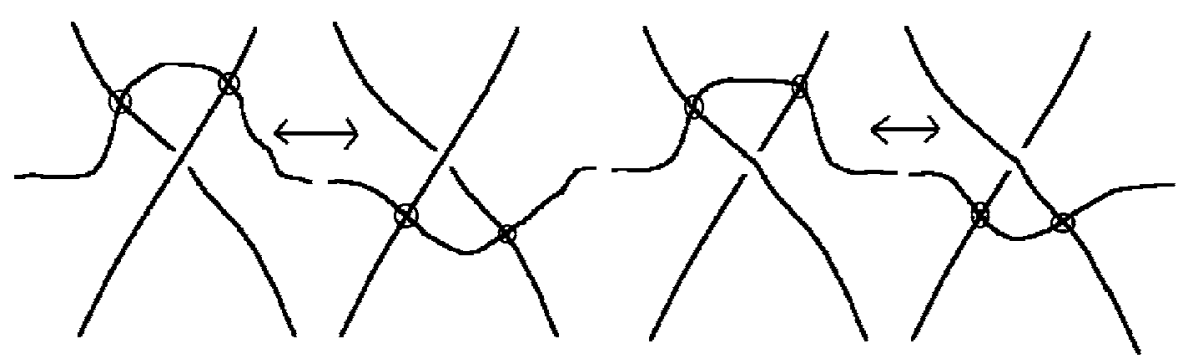

Figure 1. Semivirtual Reidemeister move.
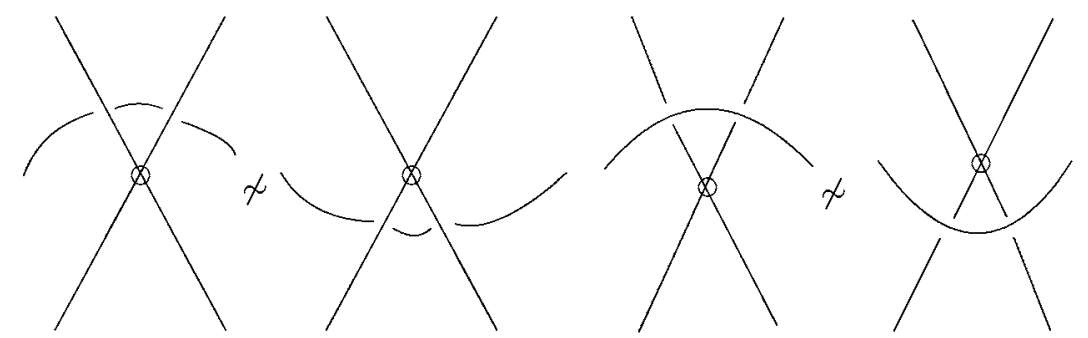

Figure 2. Forbidden Reidemeister move.

We remark that the move indicated in Figure 2 is not included in the list of virtual Reidemeister moves. In the general case it cannot be represented as a composition of generalized Reidemeister moves.

Several invariants of classical links have by now been generalized to the virtual case (see [1], [3]).

A number of virtual link invariants were constructed in [5] (there is a short version in [6]). One of them (called the $V A$-polynomial) originates from the virtual Alexander module defined in the same paper. Another strong invariant was presented in [7].

The $V A$-polynomial is a polynomial over the ring $\mathbb{Q}\left[t, t^{-1}\right]=R$. It is defined up to invertible elements of the ring $R$, that is, up to $\lambda t^{k}$, where $\lambda \in \mathbb{Q}, k \in \mathbb{Z}$. In this article we show how to define a more precise invariant polynomial $V A^{\prime}$ for virtual knots (which will be an element of $\mathbb{Z}\left[t, t^{-1}\right]=R^{\prime}$ defined up to invertible elements of the ring $R^{\prime}$ ).

We establish some properties of $V A$ - and $V A^{\prime}$-polynomials for virtual knots. Moreover, we write out a table of values of these polynomials. We then define the invariant $\mathcal{V}$ of "long virtual knots" with values in $\mathbb{Q}\left[t, t^{-1}\right]$.

\section{$\S 2$. Construction of the $V A$-Polynomial and some of its properties}

We begin with the basic definitions and introduce the main notation. Most of the basic definitions in the theory of virtual knots can be found in [4].

In this paper we assume that all diagrams of virtual links are oriented unless otherwise stated. Virtual link diagrams will be denoted by letters with overlines; for example, $\bar{L}$ will denote the diagram giving the virtual link $L$.

Definition 1. An arc of a virtual diagram will be understood to be a connected component of the set obtained from a virtual diagram by deleting the virtual crossings (at the classical crossings the undercrossing line is assumed to be cut). We say that two arcs $a, a^{\prime}$ belong to the same long arc if there exist a sequence of $\operatorname{arcs} a=a_{0}, \ldots, a_{n}=a^{\prime}$ and a sequence of virtual crossings $C_{1}, \ldots, C_{n}$ such that for $i=1, \ldots, n$ the $\operatorname{arcs} a_{i}, a_{i-1}$ 


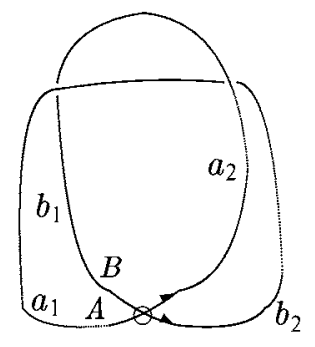

Figure 3. Arcs and long arcs of a virtual diagram.

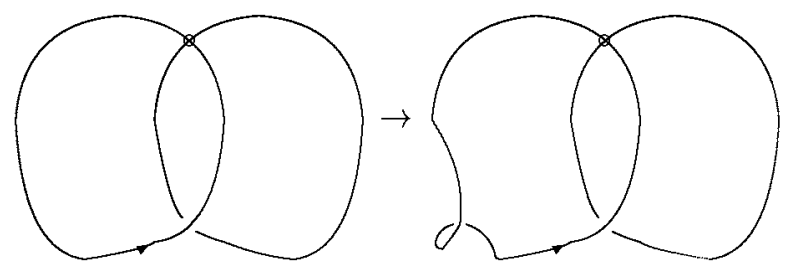

FIgURE 4. Correction of a diagram having a cyclic arc.

are incident to the vertex $C_{i}$ from opposite sides. Figure 3 shows a diagram having four arcs: $a_{1}, a_{2}, b_{1}, b_{2}$.

In what follows, long arcs will be denoted by capital Latin letters $A, B, C$ (perhaps with subscripts), and the arcs belonging to them by the same Latin letters in lower case: $a, b, c$ (also perhaps with subscripts).

For convenience all the modules in this paper will be assumed to be right-left modules.

A long arc of a virtual diagram can be cyclic, that is, it can be a closed curve, as in Figure 4,

However, this can easily be corrected: a cyclic long arc can be broken up into two noncyclic arcs by using the first classical Reidemeister move (Figure 4).

Definition 2. A virtual diagram is said to be proper if it does not have cyclic long arcs.

If a virtual diagram $\bar{L}$ is proper, then the number of its classical crossings is equal to the number of its long arcs.

We have the following simple assertion.

Assertion 1. Two proper virtual diagrams $\bar{L}_{1}$ and $\bar{L}_{2}$ represent the same link if and only if there is a sequence of virtual Reidemeister moves carrying $\bar{L}_{1}$ into $\bar{L}_{2}$ such that all intermediate diagrams are also proper.

The proof is left as an easy exercise for the reader.

In the sequel we deal only with proper virtual link diagrams.

We now proceed to the definition of the $V A$-polynomial. We first define the virtual module.

Let $\bar{L}$ be a virtual diagram with $n$ classical crossings, and denote its long arcs by $A_{1}, \ldots, A_{n}$. For each $i=1, \ldots, n$ we label all the arcs corresponding to the arc $A_{i}$ by $a_{i j}, j \in \mathbb{N}$.

Consider the ring $\mathbb{Q}\left[t, t^{-1}\right]$ and the free module $F$ over it generated by all the elements $a_{i j}$ and the formal generator $\varepsilon$.

We now factorize the module $F$ by the following relations.

At each virtual crossing there are four $\operatorname{arcs} a_{i p}, a_{i q}, a_{j r}, a_{j s}$ incident to it (some of them may coincide); see Figure 5 


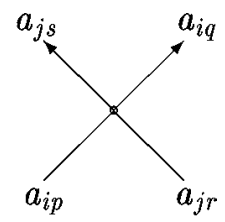

Figure 5. The arcs incident to a virtual crossing.

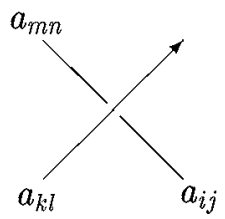

Figure 6 . The arcs at a classical crossing

We write the relations

$$
\begin{aligned}
& a_{i q}=a_{i p}+\varepsilon, \\
& a_{j s}=a_{j r}-\varepsilon .
\end{aligned}
$$

Let $F$ be factored by the set of relations of the type (1) coming from all virtual crossings, and denote the resulting factor module by $\tilde{F}$.

Three arcs meet at each classical crossing (some of them may coincide): $a_{i j}, a_{k l}, a_{m n}$, (see Figure 6).

We have not indicated any special orientation for the $\operatorname{arcs} a_{i j}$ and $a_{m n}$. In any case, we write the relation

$$
a_{i j} t+a_{k l}(1-t)=a_{m n},
$$

and factor the module $\tilde{F}$ by all relations of the form (2) for all classical crossings. Let $\mathcal{M}(\bar{L})$ denote the resulting module.

Theorem 1 ([5]). The pair (the module $\mathcal{M}$, the element $\varepsilon \in \mathcal{M})$ is a virtual link invariant, that is, for different diagrams $\bar{L}_{1}$ and $\bar{L}_{2}$ of the same virtual link $\bar{L}$ there is a module isomorphism $h: \mathcal{M}\left(L_{1}\right) \rightarrow \mathcal{M}\left(L_{2}\right)$ such that $h(\varepsilon)=\varepsilon$.

We shall prove this theorem here, because ideas analogous to those used in the proof will be needed later in the paper.

Proof. We must show how the specification of the module by generators and relations changes under Reidemeister moves, verifying that in fact the module does not change.

Consider some virtual Reidemeister move. Let $\bar{L}$ and $\bar{L}^{\prime}$ be two diagrams of some virtual link that are obtained from each other by using this move. Since the move is applied inside some small circle $C$, it follows that the arcs of the diagrams $\bar{L}$ (respectively, $\bar{L}^{\prime}$ ) can be broken up into the following sets: the common set $E$ of "external" arcs belonging to both $L$ and $L^{\prime}$, the common set $S$ of arcs intersecting the circle $C$, and the two sets $I$ and $I^{\prime}$ of internal arcs belonging to $\bar{L}$ and $\bar{L}^{\prime}$, respectively. Thus, the module $\mathcal{M}(\bar{L})$ is generated by the element $\varepsilon$ and the arcs in the families $E, S, I$, while $\mathcal{M}\left(\bar{L}^{\prime}\right)$ is generated by $\varepsilon$ and the arcs in the family $E, S, I^{\prime}$. The relations (crossings) for the diagrams $\bar{L}$ and $\bar{L}^{\prime}$ can also be broken up into families: the set $R_{E}$ of external relations which are common for $\bar{L}$ and $\bar{L}^{\prime}$, and the sets $R_{I}$ and $R_{I}^{\prime}$ of internal relations for $\bar{L}$ and $\bar{L}^{\prime}$, respectively. 


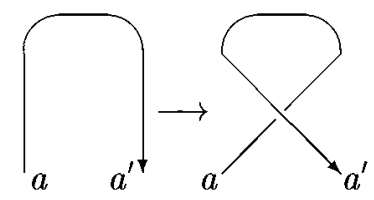

FiguRE 7 . The invariance of $(\mathcal{M}, \varepsilon)$ under $\Omega_{1}$.

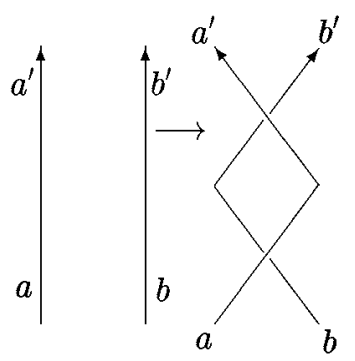

FiguRE 8. Invariance of the pair $(\mathcal{M}, \varepsilon)$ under $\Omega_{2}$.

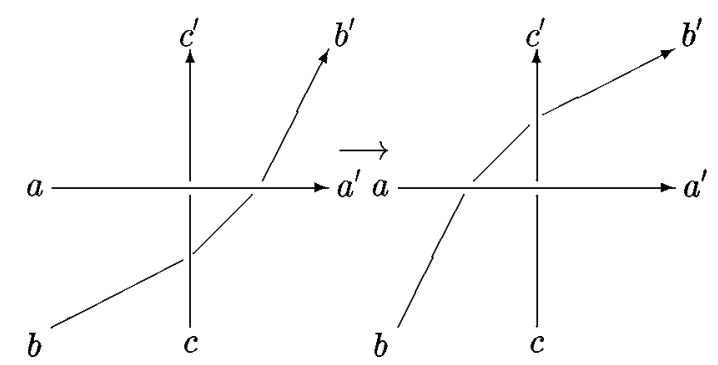

Figure 9. Invariance of the pair $(\mathcal{M}, \varepsilon)$ under $\Omega_{3}$

It is easy to see that in the case of each generalized Reidemeister move we can remove the generators in $I$, expressing them in terms of generators in $S$ with the help of the relations in $R_{I}$. Such a "removal" adds some "internal" relations in $R_{S}$ for $S$. An analogous procedure can be carried out also for $I^{\prime}$. We denote by $R_{S}^{\prime}$ the set of relations thereby obtained. Thus, we have transformed the two modules $\mathcal{M}(\bar{L})$ and $\mathcal{M}\left(\bar{L}^{\prime}\right)$ into modules $\tilde{\mathcal{M}}(\bar{L})$ and $\tilde{\mathcal{M}}\left(\bar{L}^{\prime}\right)$ isomorphic to them. The resulting modules are generated by only the generators in $E$ and $S$ (and $\varepsilon$ ). They have a common set $R_{E}$ of "external" relations. Thus, it remains to show that the relations in $R_{S}$ and $R_{S}^{\prime}$ give the same equivalence relation on $S$ (by means of $\varepsilon$ ).

For each of the virtual Reidemeister moves we consider one concrete version; all the remaining versions can be treated in a completely similar way.

The first classical Reidemeister move gives the relation $a^{\prime}=a$ in both cases: for $R_{S}$ and for $R_{S}^{\prime}$ (see Figure 7).

The second classical Reidemeister move gives the relations $a^{\prime}=a$ and $b^{\prime}=b$ in both cases (Figure 8).

Finally, the third classical Reidemeister move (Figure 9) gives the relations

$$
a^{\prime}=a, \quad c^{\prime}=c t+a(1-t), \quad b^{\prime}=(b t+c(1-t)) t+a(1-t)
$$




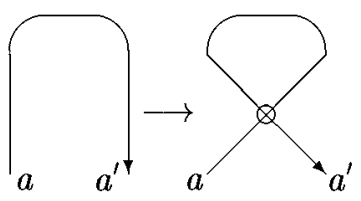

FiguRe 10. Invariance of the pair $(\mathcal{M}, \varepsilon)$ under the first virtual move.

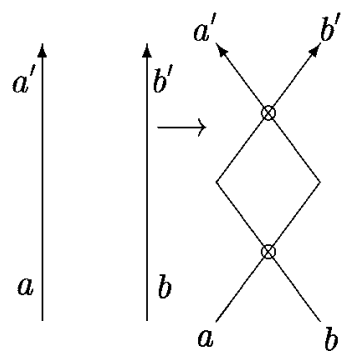

FiguRE 11. Invariance of the pair $(\mathcal{M}, \varepsilon)$ under the second virtual move.

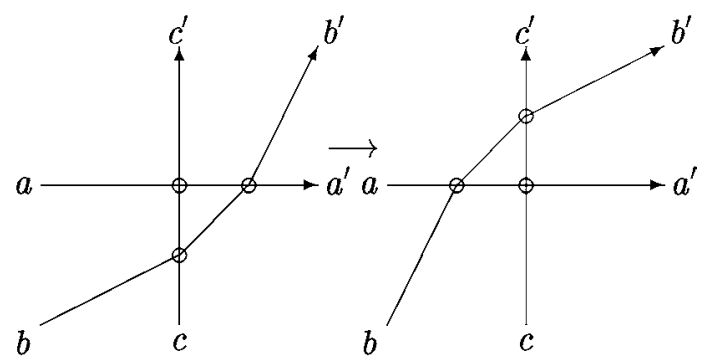

FIGURE 12. Invariance of the pair $(\mathcal{M}, \varepsilon)$ under the third virtual move.

in the case $R_{S}$ and the relations

$$
a^{\prime}=a, \quad c^{\prime}=c t+a(1-t), \quad b^{\prime}=(b t+a(1-t)) t+(c t+a(1-t))(1-t)
$$

in the case $R_{S}^{\prime}$.

It is seen that the expression of $c^{\prime}$ in terms of $a, b, c$ is the same in both cases.

We now proceed to the purely virtual Reidemeister moves.

The first purely virtual Reidemeister move gives the relation $a=a^{\prime}$ in both cases, as shown in Figure 10 .

The second virtual move gives the relations $a=a^{\prime}$ and $b=b^{\prime}$ in both cases $R_{S}$ and $R_{S}^{\prime}$ (Figure 11).

The third virtual move is shown in Figure 12. Both sets $R_{S}$ and $R_{S^{\prime}}$ thus contain the relations $a^{\prime}=a+2 \varepsilon, b^{\prime}=b$, and $c^{\prime}=c-2 \varepsilon$.

The case of a mixed move is pictured in Figure 13. The set $R_{S}$ consists of the relations $a^{\prime}=a+\varepsilon, b^{\prime}=b$, and $c^{\prime}=(c-\varepsilon) t+a(1-t)$ in this case, and the set $R_{S}$ consists of the relations $a^{\prime}=a+\varepsilon, b^{\prime}=b$, and $c^{\prime}=c t+(a+\varepsilon)(1-t)-\varepsilon$.

Thus, $R$ and $R^{\prime}$ really do give the same.

We have considered all the generalized Reidemeister moves. The theorem is thereby proved.

Together with the pair $(\mathcal{M}, \varepsilon)$, any characteristic associated with it is also invariant. 


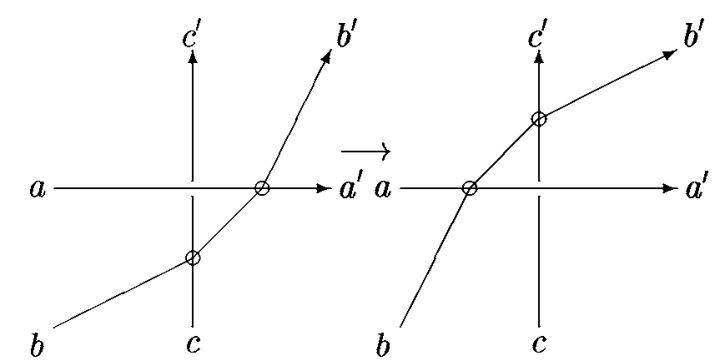

FiguRE 13. Invariance of the pair $(\mathcal{M}, \varepsilon)$ under a mixed move.

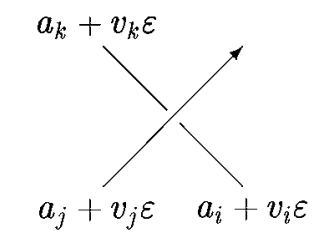

FIGURE 14. Rewriting of the relations for classical crossings.

Consider a link $L$ and compute the pair $(\mathcal{M}, \varepsilon)$ for it. Denote by $I(\mathcal{M}, \varepsilon)$ the ideal of $R$ generated by all $i \in R$ for which $i \varepsilon=0$. The ring $R$ is a Euclidean ring, and thus the ideal $I$ is principal. Consequently, it is generated by some element $x$ which is determined up to invertible elements of $R$. If the ideal consists solely of 0 , then we take $x=0$ by definition. By construction, this element $x$ is an invariant of the link $L$.

Definition 3. We denote by $V A(L)$ the element $x$ obtained and call it the VA-polynomial.

\section{$\S 3$. How is the $V A$-POlynomial COMPuted?}

We proceed to a simplification of the method for constructing the module $\mathcal{M}$. Namely, for a given link diagram $\bar{L}$ with $n$ long arcs we consider all the $\operatorname{arcs} a_{11}, \ldots, a_{n 1}$. It is obvious that for each $k$ we have $a_{i k}=a_{i 1}+P_{i k} \varepsilon$, where $P_{p q}$ are integers. Here we use the fact that the diagram $\bar{L}$ is proper, and all the $P_{i k}$ are well defined.

Thus, we can take only $a_{i 1}$ and $\varepsilon$ as generators for $M(\bar{L})$.

Three arcs meet at each classical crossing (see Figure 14).

Next we write out the relation

$$
\left(a_{i}+v_{i} \varepsilon\right) t+\left(a_{j}+v_{j} \varepsilon\right)(1-t)=a_{k}+v_{k} \varepsilon
$$

or, transposing all the terms containing $\varepsilon$ to the right-hand side,

$$
t \cdot a_{i}+(1-t) a_{j}-a_{k}=\varepsilon \cdot(\text { an integer }) .
$$

We recall that the number of classical crossings of our diagram is equal to $n$. If a crossing is negative, then we multiply both sides of (4) by -1 . Thus, our module is given by a system of $n$ equations with $n+1$ variables. We call the column of coefficients of $\varepsilon$ on the right-hand side the $\varepsilon$-column.

Considering only the left-hand sides of these relations (concerning $a_{1}, \ldots, a_{n}$ ), we obtain a matrix.

Definition 4. This matrix will be called the virtual Alexander matrix of the diagram $\bar{L}$ and denoted by $M(\bar{L})$. 
Obviously, the sum of the elements in each row of the matrix $M(\bar{L})$ is zero. Thus, the rows are linearly dependent. All possible linear dependences on the left-hand side of (4) give certain relations on the right-hand side. They thereby generate the elements of the desired ideal.

We prove the following lemma.

Lemma 1. For the diagram $\bar{L}_{m}$ of an m-component virtual link the corank of the virtual Alexander matrix is at most $m$.

Proof. It suffices to prove that the value of the matrix $M\left(\bar{L}_{m}\right)$ for $t=1$ has corank $m$.

Indeed, each row of this matrix consists of two nonzero elements: 1 and -1 . They correspond to the two long arcs incident to a given crossing (incoming and outgoing). Thus, our matrix breaks up into blocks corresponding to the components of the link. After a preliminary renumbering of the rows and columns and after (possibly) multiplication of some of the rows by -1 , each matrix block will have the form

$$
\left(\begin{array}{rrrrrr}
1 & -1 & 0 & 0 & \ldots & 0 \\
0 & 1 & -1 & 0 & \ldots & 0 \\
\vdots & 0 & \ddots & \ddots & 0 & \vdots \\
0 & \ldots & 0 & 1 & -1 & 0 \\
0 & \ldots & 0 & 0 & 1 & -1 \\
-1 & 0 & 0 & \ldots & 0 & 1
\end{array}\right) .
$$

Thus, each block has corank 1, which implies that the matrix has corank $m$.

One can now get the value of the $V A$-polynomial directly from the matrix: just find all the linear dependences of the rows.

\section{$\S 4$. TABle of Values of the $V A$-POLYNOMial}

Table 1 gives the values of the $V A$-polynomial on some virtual knots and links. In particular, we included some virtual knots not isotopic to classical knots, on which the $V A$-polynomial is equal to zero.

Remark 1 . The fact that on classical links the $V A$-polynomial is equal to zero was proved in [5].

\section{$\S 5$. Some Properties of the $V A$-Polynomial}

Theorem 2. For each virtual knot $K$ (not link) the value $V A(K)$ is divisible by $(t-1)^{2}$.

Proof. Let $\bar{K}$ be the diagram of a virtual knot. In the case when all its crossings are virtual, $\bar{K}$ is a diagram of the trivial knot and has $V A$-polynomial equal to zero. Otherwise, we consider a classical crossing $V_{1}$ of the diagram $\bar{K}$. Let $X$ be a long $\operatorname{arc}$ going out from $V_{1}$ and let $x$ be the first arc of $X$ incident to $V_{1}$ (with respect to the orientation of the knot). Denote this arc by $a_{1}$. By construction, to all the arcs belonging to the long arc $X$ there correspond numbers $a_{1}+k \varepsilon, k \in \mathbb{N}$. Suppose that the last (along the orientation of the knot) long arc of the $\operatorname{arc} X$ is labeled by $a_{1}+k_{1} \varepsilon$. Denote the final crossing of this arc by $V_{2}$. We now consider the first arc going out from $V_{2}$ and assign $a_{2}+k_{1} \varepsilon$ to it. Next, we set labels of the form $a_{2}+k \varepsilon$ for all the arcs belonging to the same long arc. Assume that the last of them has the label $a_{2}+k_{2} \varepsilon$ and ends at the crossing $V_{3}$. We then assign the label $a_{3}+k_{2}$ to the first arc going out from $V_{3}$, and so on.

We finally come to the crossing $V_{1}$. Let us prove that the process converges, that is, the label we should assign to the arc first going out from $V_{1}$ must be $a_{j+1}+0 \cdot \varepsilon$, where $j$ is the total number of long arcs. 
TABLE 1 . The values of the $V A$-polynomial.

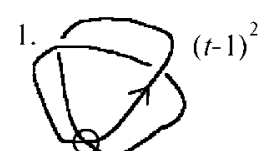

4.

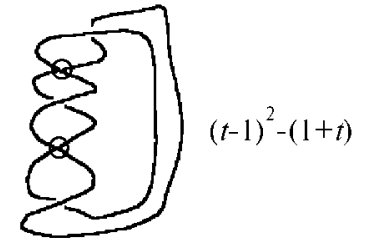

6

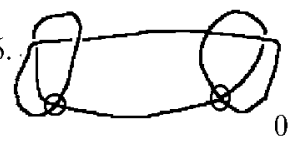

8.

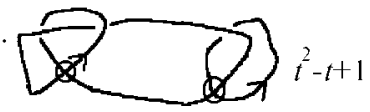

10
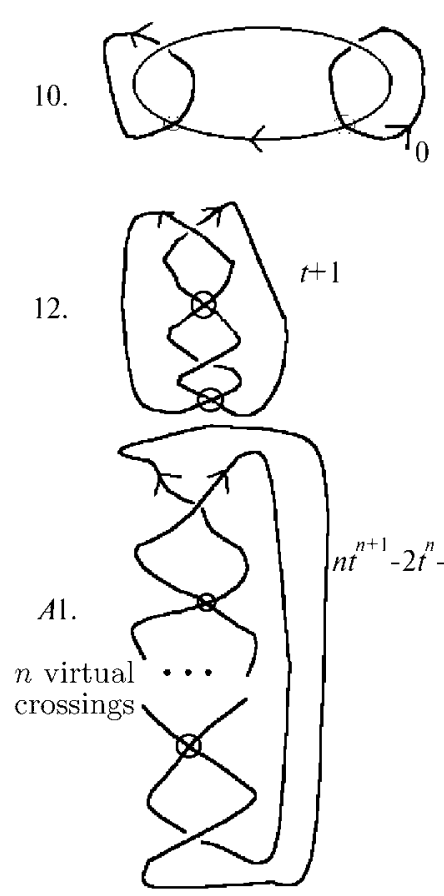

2.

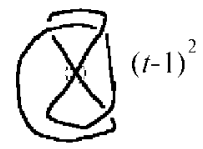

5.

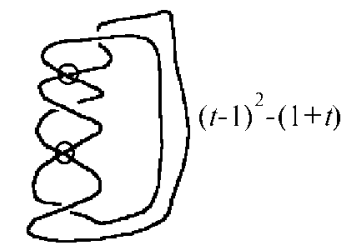

7.

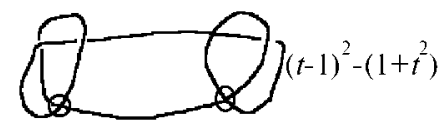

9.

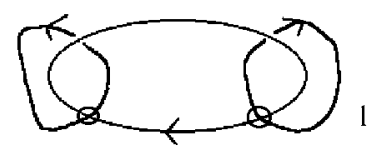

11.

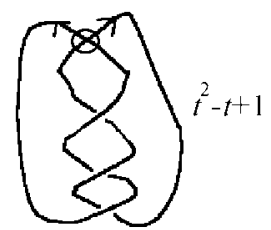

13.
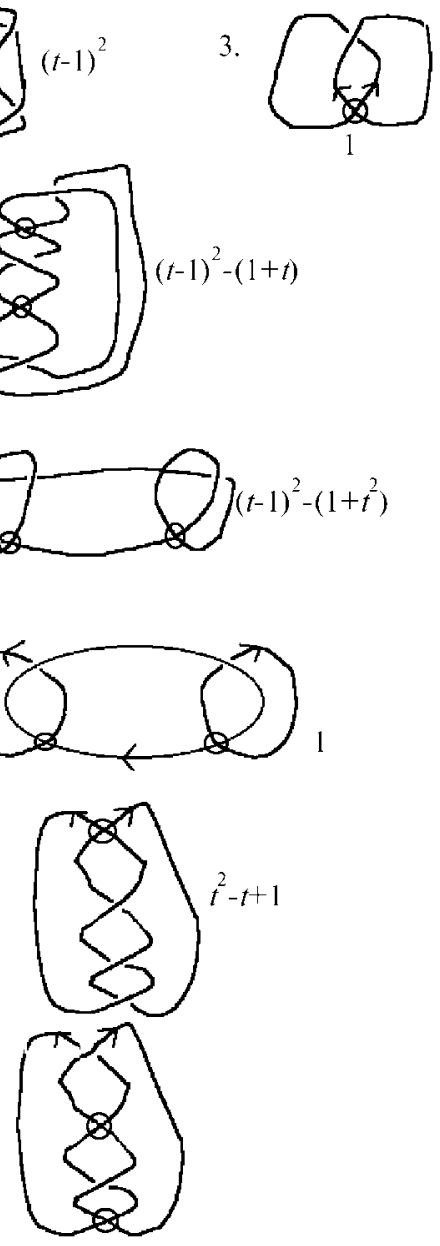
We remark that the process converges if we proceed in the same way starting at any arc with any prescribed label.

In this case each relation in the virtual Alexander module has a right-hand side divisible by $\varepsilon(t-1)$, since the relation

$$
\left(a_{i}+p \varepsilon\right) t+\left(a_{j}+q \varepsilon\right)(1-t)=\left(a_{k}+p \varepsilon\right)
$$

is equivalent to the relation

$$
a_{i} t+a_{j}(1-t)-a_{k}=(t-1)(q-p) \varepsilon .
$$

This proves that $V A(K)$ is divisible by $(t-1)$.

Denote the elements $q$ and $p$ on the right-hand side of the equation $(*)$ corresponding to the $i$ th vertex by $q_{i}$ and $p_{i}$, respectively.

We look for relations on the rows of the virtual Alexander matrix. Each relation must hold for any $t$, and thus also for $t=1$.

Let the rows of the matrix $M$ be denoted by $M_{i}$. If for $M$ we have

$$
\sum_{i=1}^{n} c_{i} M_{i}=0
$$

then

$$
\left.\left.\sum_{i=1}^{n} c_{i}\right|_{t=1} M_{i}\right|_{t=1}=0 .
$$

The matrix $\left.M(\bar{K})\right|_{t=1}$ looks very simple. Each of its rows (and each of its columns) contains a single 1 and a single -1 , with the rest of the elements equal to 0 . The relation on the rows of the matrix is obvious: the sum of them all is equal to 0 . Thus, $\left.c_{i}\right|_{t=1}=\left.c_{j}\right|_{t=1}$ for all $i, j=1, \ldots, n$.

Each relation for $\varepsilon$ has the form

$$
\left(\sum_{i=1}^{n} c_{i}\left(q_{i}-p_{i}\right)\right)(t-1)=0 .
$$

In considering the question of divisibility of the left-hand side by $(t-1)^{2}$ we can take $(t-1)$ out of the parentheses and in the remaining sum replace all the $c_{i}$ by $\left.c_{i}\right|_{t=1}$.

Consequently, it suffices to show that

$$
\sum_{i=1}^{n}\left(q_{i}-p_{i}\right)=0
$$

for the given diagram $\bar{K}$.

We prove this fact by induction on the number $n$ of classical crossings.

Recall that a crossing is said to be positive when the undercrossing edge is oriented from left to right with respect to the overcrossing edge; otherwise, the crossing is said to be negative.

There is nothing to prove in the case $n=0$.

Suppose now that $\bar{K}$ is a diagram with $n$ classical crossings, and $\bar{K}^{\prime}$ is the diagram obtained from $\bar{K}$ by replacing one classical crossing by a virtual crossing. We consider only the case when the replaced crossing $X$ is positive (treatment of the "negative" case is similar); see Figure 15

Let the lower left-hand arc in both diagrams be denoted by $a$, and the remaining arcs by $b, c$, and $d(a=d$ for the diagram $\bar{K})$; see Figure 15. We assign the label zero to the $\operatorname{arc} a$ in both diagrams. Let us compute the sum $\sum\left(q_{i}-p_{i}\right)$ for the cases $\bar{K}^{\prime}$ and $\bar{K}$. By the induction hypothesis, it is zero for $\bar{K}^{\prime}$. 


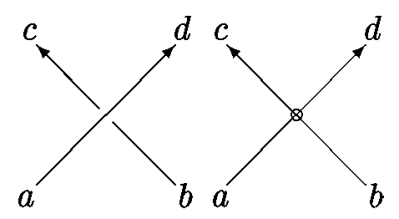

Figure 15. Labels on $\bar{K}$ and $\bar{K}^{\prime}$.

We denote the label on the arc $b$ in the first diagram by $l_{b 1}$, and in the second diagram by $l_{b 2}$.

For the crossing $X$ in $\bar{K}$ we have $q=l_{b 1}$ and $p=0$. Thus, its contribution to the total sum is equal to $l_{b 1}$.

The remaining crossings in $\bar{K}$ are in a natural one-to-one correspondence with the crossings in the diagram $\bar{K}^{\prime}$. Let us compute the difference between the numbers $p$ and $q$ for the two diagrams. This difference comes from the classical crossings. Their labels differ only on the part of the diagram between $d$ and $b$ (along the orientation of the knot). In moving from $d$ to $b$, we encounter classical and virtual crossings. The total algebraic number of these crossings is obviously equal to zero. The algebraic number of virtual crossings is equal to $(-q)$. Hence, the algebraic number of classical crossings is equal to $q$. Each of them gives a contribution of -1 to the difference between $\bar{K}$ and $\bar{K}^{\prime}$. Therefore, we have $q-q=0$; this concludes the induction step, and thus proves the theorem.

The following theorem is obvious.

Theorem 3. The invariant $V A$ is additive in the sense that for any connected sum $K=K_{1} \# K_{2}$ of two links $K_{1}$ and $K_{2}$ there exist invertible elements $\lambda, \mu \in R$ such that $V A(K)=\lambda V A\left(K_{1}\right)+\mu V A\left(K_{2}\right)$.

\section{$\S 6$. THE CASE OF $\mathbb{Z}\left[t, t^{-1}\right]$}

At the beginning of the paper we considered $\mathbb{Q}\left[t, t^{-1}\right]$ because it is a Euclidean ring. However, we can also consider the ring $\mathbb{Z}\left[t, t^{-1}\right]$ with the goal of defining an invariant polynomial (an analogue of the $V A$-polynomial) that is more precise. In the new ring the set of elements under consideration is smaller. Thus, the element giving the principal ideal can be determined more precisely. On the other hand, not every ideal in $\mathbb{Z}\left[t, t^{-1}\right]$ is principal. For example, the ideal $\{2, t+1\}$ is not principal.

Notation: In this section $R=\mathbb{Z}\left[t, t^{-1}\right]$, all modules are over $R$, and all (Laurent) polynomials are assumed to have integer coefficients. For simplicity of notation we use the same letters $\mathcal{M}, \varepsilon$ for the objects analogous to those introduced above. The polynomial we are going to define will be denoted by $V A^{\prime}$.

Consider a virtual link $L$ and a diagram $\bar{L}$ of it. We construct a virtual Alexander module over $R$ for this diagram just as we did over $\mathbb{Q}\left[t, t^{-1}\right]$.

Arguing as before, we can prove the following theorem.

Theorem 4. The virtual Alexander module $\mathcal{M}$ over $R$ together with the distinguished element $\varepsilon$ is an invariant with respect to generalized Reidemeister moves.

Thus, we can construct $(\mathcal{M}, \varepsilon)(L)$ for any virtual link $L$.

As before, we can define the ideal $I$ corresponding to $L$ to be the set of elements $i \in R$ for which $i \varepsilon=0$.

By definition, this ideal $I(L)$ is an invariant of virtual links. However, it can in general fail to be a principal ideal, since $R$ is not a Euclidean ring.

We have the following theorem. 
Theorem 5. For each virtual knot (not link) $K$ the ideal $I(K)$ is principal.

Proof. Consider a diagram $\bar{K}$ of the knot $K$ and write out the virtual Alexander matrix $M$ for it. By Lemma 1 (the proof of which is literally the same for both rings), the corank of $M$ is at most 1. Since the matrix is singular, this corank is precisely 1 .

Thus, the rank of our Alexander matrix of $n$ rows is equal to $n-1$.

We denote the rows of this matrix by $m_{1}, \ldots, m_{n}$. Let $\left(\alpha_{1}, \ldots, \alpha_{n}\right)$ and $\left(\beta_{1}, \ldots, \beta_{n}\right)$ be two sets of elements of $R$ such that

$$
\sum_{i=1}^{n} \alpha_{i} m_{i}=\sum_{i=1}^{n} \beta_{i} m_{i}=0
$$

In this case the sets are proportional, that is, there exist $p, q \in R$ such that $p \alpha_{i}=q \beta_{i}$ for any $i=1, \ldots, n$. Indeed, if we consider exactly the same matrix over the field $\mathbb{Q}(t)$, then it will have rank $n-1$. Therefore, each two eigenvectors with zero eigenvalue are proportional over this field. Denoting the element expressing this proportionality by $\frac{p}{q}$, $p, q \in R$, we obtain the desired assertion.

Now let $\alpha$ be the greatest common divisor of the elements $\alpha_{1}, \ldots, \alpha_{n}$ in $R$. It is determined up to elements invertible in $R$.

Let $\frac{\alpha_{i}}{\alpha}$ be denoted by $\alpha_{i}^{\prime}$. Obviously, $\sum_{i=1}^{n} \alpha_{i}^{\prime}=0$. Thus, for any set $\left(\gamma_{1}, \ldots, \gamma_{n}\right)$ with $\sum_{i=1}^{n} \gamma_{i} m_{i}=0$ there is a $\gamma$ such that $\gamma_{i}=\gamma \alpha_{i}$ for all $i=1, \ldots, n$, because the set of $\gamma_{i}$ is proportional to the set of $\alpha_{i}^{\prime}$, while the elements of the last set are relatively prime in $R$.

We now consider the $\varepsilon$-column $\left(b_{1}, \ldots, b_{n}\right)$. The linear dependence coming from $\left(\alpha_{1}^{\prime}, \ldots, \alpha_{n}^{\prime}\right)$ implies the relation $i \varepsilon=0$, where $i=\sum_{j=1}^{n} \alpha_{j}^{\prime} b_{j}$.

As noted above, any other $n$-tuple $\left(\gamma_{1}, \ldots, \gamma_{n}\right)$ giving some linear dependence can be obtained from the row $\left(\alpha_{1}^{\prime}, \ldots, \alpha_{n}^{\prime}\right)$ by multiplying it by an element of $R$. Consequently, the element $i$ is also multiplied by an element of $R$.

Therefore, the ideal $I(K)$ is generated by $i$.

We have thereby defined a polynomial that is more precise that $V A$; denote it by $V A^{\prime}$.

For example, if we take a "virtual trefoil" (Figure 16a) and its connected sum with itself (Figure 16]b), then the $V A$-polynomials obtained give the same result, whereas the $V A^{\prime}$-polynomial for the knot in Figure 16]a is equal to $(t-1)^{2}$, while for Figure 16 b it is equal to $2(t-1)^{2}$.

By similar methods it is possible to construct an invariant polynomial in one variable for virtual links that coincides with the Alexander polynomial on classical links.

Namely, let $\bar{L}$ be a proper diagram of a virtual link, and let $M(L)$ be its Alexander matrix. Denote by $\mathcal{M}(L)$ the Alexander module constructed in a similar way without $\varepsilon$. In the classical case the Alexander polynomial $\Delta$ is defined as a minor of the corresponding matrix (the minors all coincide up to multiplication by $\pm t^{k}$ for some $k$ ). We consider all the long $\operatorname{arcs} a_{1}, \ldots, a_{n}$ of the diagram $\bar{L}$. Let $\mu$ be the linear space of vectors (in the basis $a_{1}, \ldots, a_{n}$ ) with sum of coordinates zero.

It is easy to verify that $\mu$ is an invariant subspace of $M$ with respect to generalized Reidemeister moves.

We denote by $\Delta(\bar{L})$ the minimal element in $\mathbb{Q}\left[t, t^{-1}\right]$ such that $\Delta \cdot x=0$ for all $x \in \mu$.

The next theorem follows at once from the definition.

Theorem 6. The polynomial $\Delta(\bar{L})$ constructed is a virtual link invariant up to multiplication by $\mathbb{Q}\left[t, t^{-1}\right]$.

Thus, we can write $\Delta(L)$. It is easy to see that this polynomial coincides with the Alexander polynomial on diagrams of classical links. 
a)

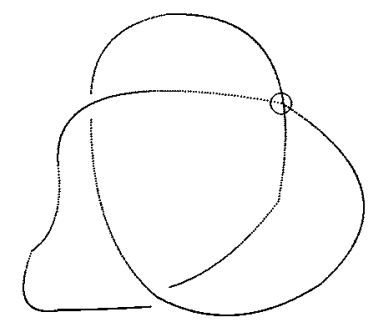

b)

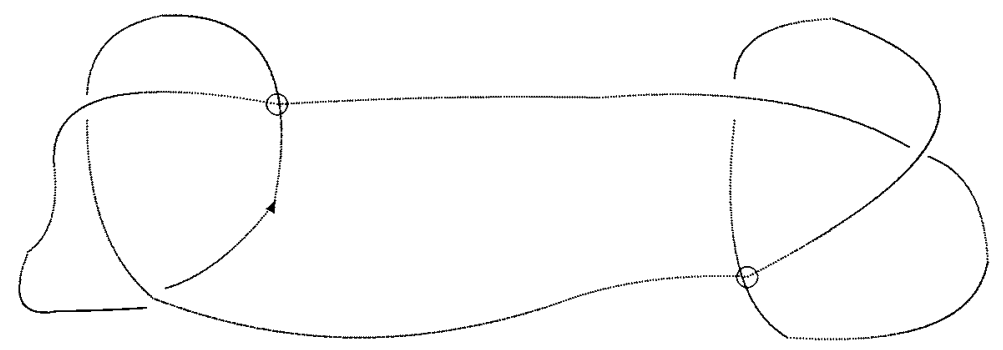

Figure 16. A virtual trefoil and its connected sum with itself.

\section{$\S 7$. The Polynomial $\mathcal{V}$}

As shown in [5], the procedure for cutting a virtual knot is not well defined. For example, by cutting the same virtual knot at different points we obtain different (not isotopic) long knots. The purpose of this section is to construct an invariant of long virtual knots.

Throughout this section, $R$ will denote the field $R=\mathbb{Q}(t)$ of rational functions.

Definition 5. A diagram of a long virtual knot will be understood to be a smooth immersion $f$ of an oriented line $L_{t}, t \in(-\infty,+\infty)$, in $\mathbb{R}^{2}$ such that:

1) $f(t)=(t, 0)$ outside a large disk;

2) each self-intersection point of this immersion is simple and transversal;

3 ) each intersection point is equipped with the structure of a classical or virtual crossing.

Definition 6. A long virtual knot is an equivalence class of diagrams of long virtual knots modulo generalized Reidemeister moves.

Obviously, given a diagram of a virtual knot, we can cut it at some "external" point in order to get a diagram of a long virtual knot (Figure 17).

It is generally known that in the case of ordinary knots the result (that is, the isotopy class of the resulting long knot) does not depend on the choice of the cut point.

We show that this is not so in the virtual case. Namely, we use ideas described earlier to construct an invariant of long virtual links which, in particular, proves this fact.

By an arc and a long arc we understand exactly the same as in the classical case. Each diagram has two special arcs, namely, the arcs going to infinity.

Consider a diagram $\bar{K}$ of a long knot $K$. We construct the virtual Alexander module for it just as before. For simplicity we keep the previous notation. This module (over $R$ ) is denoted by $\mathcal{M}$.

Suppose that we have $n+1$ long arcs (this corresponds to $n$ long arcs in the classical case and $n$ crossings). Each of the two infinite long arcs has one infinite arc. We denote the arc containing $-\infty$ by $a_{1}$, and the arc containing $+\infty$ by $a_{n+1}$. For each of the 

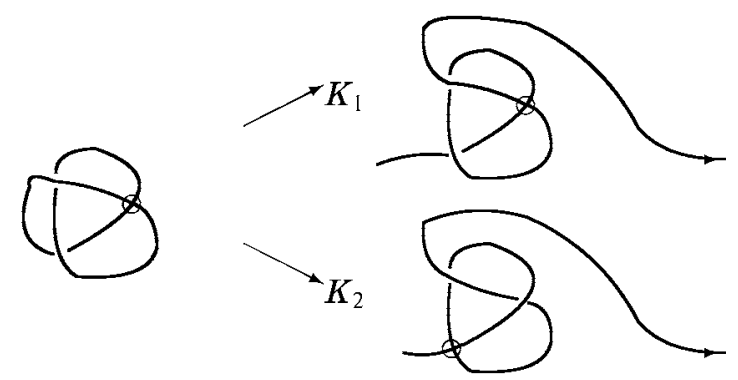

Figure 17. Transformation of a diagram into a "long" diagram.

remaining $n-1$ long arcs we choose one short arc belonging to it. Denote these arcs by $a_{2}, \ldots, a_{n}$.

We construct a linear space over $R$. First, we consider the $(n+2)$-dimensional space $S$ generated by the elements $a_{1}, \ldots, a_{n+1}, \varepsilon$.

We now define $\mathcal{M}$ as the quotient space of $S$ with respect to the relations just as in the classical case. Then we obtain an invariant triple $\left(\mathcal{M}, a_{1}, a_{n+1}\right)$. Obviously, $a_{n+1}-a_{1}=k \cdot \varepsilon$.

By definition, this $k$ is an invariant of long knots. Denote it by $\mathcal{V}(K)$.

The following property of the $\mathcal{V}$-polynomial is obvious.

Theorem 7. $\mathcal{V}(L)=0$ for any classical long knot $L$.

Consider now the following example. In [5] it was shown that on cutting a virtual trefoil in two different ways (Figure 17) one obtains two different long virtual knots. This was proved by using the $V A$-polynomial on connected sums of virtual knots. We now prove it with the help of the polynomial $\mathcal{V}$.

For the knot $K_{1}$ we have

$$
\begin{aligned}
& a_{1}(-t)+a_{2} t-a_{3}=\varepsilon(-2 t), \\
& a_{1} t+a_{3}(-t)-a_{2}=\varepsilon t .
\end{aligned}
$$

Multiplying the second equation by $t$ and adding it to the first, we get that

$$
\mathcal{V}\left(K_{1}\right)=a_{3}-a_{1}=-\frac{t^{2}-t+1}{(t-1)^{2}}
$$

For $K_{2}$ we have

$$
\begin{array}{r}
t a_{1}+(-t)\left(a_{3}+\varepsilon\right)-a_{2}=0, \\
t\left(a_{2}+\varepsilon\right)+(-t) a_{3}-\left(a_{3}+\varepsilon\right)=0 .
\end{array}
$$

Multiplying the first equation by $t$ and adding it to the second, we get that

$$
\mathcal{V}\left(K_{2}\right)=-\frac{t^{2}}{(t-1)^{2}} .
$$

Thus, $\mathcal{V}$ distinguishes long virtual knots coming from the same virtual knot.

Taking a connected sum \# of long virtual knots is a well-defined operation.

The following theorem is obvious.

Theorem 8. For any two virtual knots $K_{1}$ and $K_{2}$

$$
\mathcal{V}\left(K_{1} \# K_{2}\right)=\mathcal{V}\left(K_{1}\right)+\mathcal{V}\left(K_{2}\right)
$$




\section{REFERENCES}

[1] M. Goussarov, M. Polyak, and O. Viro, Finite-type invariants of classical and virtual knots, Topology 39 (2000), 1045-1068. MF,1763963 (2001i:57017)

[2] F. Jaeger, L. H. Kauffman, and H. Saleur, The Conway polynomial in $S^{3}$ and thickened surfaces: a new determinant formulation, J. Combin. Theory Ser. B 61 (1994), 237-259. MR1280610 (95d:57004)

[3] L. H. Kauffman, Virtual knot theory, European J. Combin. 20 (1999), 662-690. MR1721925 (2000i:57011)

[4] V. O. Manturov, Lectures on the theory of knots and their invariants, URSS, Moscow, 2001; English transl., Knot theory, CRC Press, London, 2004.

[5] V. O. Manturov, On invariants of virtual links, Acta Appl. Math. 72 (2002), no. 3, 295-309. MR1916950 (2004d:57010)

[6] V. O. Manturov, Invariants of virtual links, Dokl. Ross. Akad. Nauk Ser. Mat. 384 (2002), 11-13; English transl. in Doklady Math. 65 (2002), 329-331. MR.1932200 (2003m:57033)

[7] V. O. Manturov, Curves on surfaces, virtual knots, and the Jones-Kauffman polynomial, Dokl. Ross. Akad. Nauk Ser. Mat. 390 (2003), 155-157; English transl. in Doklady Math. 67 (2003), 326-328. MR2003612

Moscow State University, Mechanics and Mathematics Department, 119899 Moscow, Russia

E-mail address: vassily@manturov.mccme.ru

Translated by H. H. MCFADEN 